\title{
Structure Design and Analysis of a Compact Breast Biopsy Robot under MRI Environment
}

\author{
Yongde Zhang ${ }^{1}$, Haiyan $\mathrm{Du}^{1}$, Yuqin $\mathrm{Li}^{1}$ and Hongxia Zhang ${ }^{2}$ \\ ${ }^{1}$ Intelligent Machine Institute, Harbin University of Science and Technology, Harbin \\ 150080, China \\ ${ }^{2}$ Harbin Medical University Cancer Hospital, Harbin 150081, China \\ zhangyd@hrbust.edu.cn,duhaiyan999@163.com
}

\begin{abstract}
Because of its excellent image quality, magnetic resonance imaging (MRI), as a new means of image guidance for breast biopsy robot, is used widely in clinical diagnostics and advanced breast research. However, the robot for MRI-guided biopsy is challenging due to the compatibility caused by strong magnetic field and very limited available space inside MR scanner. In this paper, a five degrees of freedom (DOF) robot is presented to perform biopsy in the breast with MRI guidance for the early detection of breast cancer. In an MR scanner (1.5T), the compatibility of copper, A4 non-magnetic stainless steel and POM, nylon, teflon is tested, and analyze the influence on the image of water model. Based on the analysis of available space, a 5 DOF robot which consists of 3-DOF Cartesian motions, 1-DOF rotation and 1-DOF insertion is designed, and the statics analysis of the robot is simulated using ANSYS. The results of statics analysis demonstrate that the robot meet the requirement of stiffness and precision.
\end{abstract}

Keywords: medical robotics, breast biopsy, magnetic resonance imaging

\section{Introduction}

Breast cancer is by far the most common cause of cancer mortality in women worldwide, accounting for $16 \%$ of cancer deaths in adult women [1]. Currently there is not sufficient knowledge on the causes of breast cancer; therefore, early detection and treatment of breast cancer are major factors for effective patient management. When breast cancer is detected early, and if adequate diagnosis and treatment are available, there is a good chance that breast cancer can be cured. The current treatment for breast cancer focuses on minimally invasive technique instead of the traditional surgical therapies. New minimally invasive procedures such as percutaneous core needle biopsy, interstitial laser ablation, radiofrequency ablation, and cryotherapy may provide treatment options that are psychologically and cosmetically more acceptable to patients than traditional surgical therapies [2]. These procedures also have many benefits such as low morbidity, decreased cost, short operating time, quick recovery of patient, etc.

In medical fields, it has been generally recognized that robot-assisted biopsy surgery has more advantages in positioning accuracy and dexterity [3]. In aspect of image guidance, MRI offers a very high image quality because it can provide images with multi-dimension, multiplane and multi-parameter. Therefore, robot-assisted breast biopsy with MRI guidance has been evaluated as a robust approach for the diagnosis of breast cancer. However in designing this surgical robotic system we are faced with the problems of strong permanent magnetic 
field and very limit available space [4]. Therefore, the design of robot for breast biopsy under MRI environment is challenging.

\section{Testing and Selection of Materials}

Due to the extremely high magnetic fields used in MR scanner, traditional robot composed of ferromagnetic material cannot be used [5], otherwise eddy currents will be generated and affect the clarity of local image or cause image distortion; so great care must be taken in material selection. Specifically, nonmagnetic materials must be used to avoid forces on the robot itself. In addition, the materials near the region of interest should be non-conducting to avoid the generation of eddy currents. Eddy currents can distort the MR images and arise when the fast switching magnetic field gradients induce electric currents on the conductive parts of a device that are inside the scanner. Eddy currents may also cause undesired resistive heating of conductive objects [6].

In general, ferrous materials such as iron, steel and copper are magnetic material, have a greater impact on MRI imaging and do not meet the requirement of compatibility. While nonmagnetic stainless steel, aluminum, platinum, titanium, copper alloy, ceramic, plastic, and other paramagnetic materials can meet the compatibility requirement. We place copper, A4 non-magnetic stainless steel and POM, nylon, teflon into an MR scanner (1.5T) to test the compatibility through analyzing the influence on the image of water model. It can be seen from Figure 1 that the image of water model is seriously affected by copper; a large area of image is deficient and formed a large blind spot of magnetic resonance scanners. So copper is not suitable to be selected as the materials for the robot; Non-magnetic stainless steel can affect the brightness of the image of water mold to a certain extent, but the basic image does not appear blind spot. Taking into account the mechanical properties and MR compatibility, non-magnetic stainless steel can be a suitable material if we take some measures of magnetic shield; Engineering plastics is an ideal paramagnetic material compatible with MRI because it has no effect on the image clarity of water model.
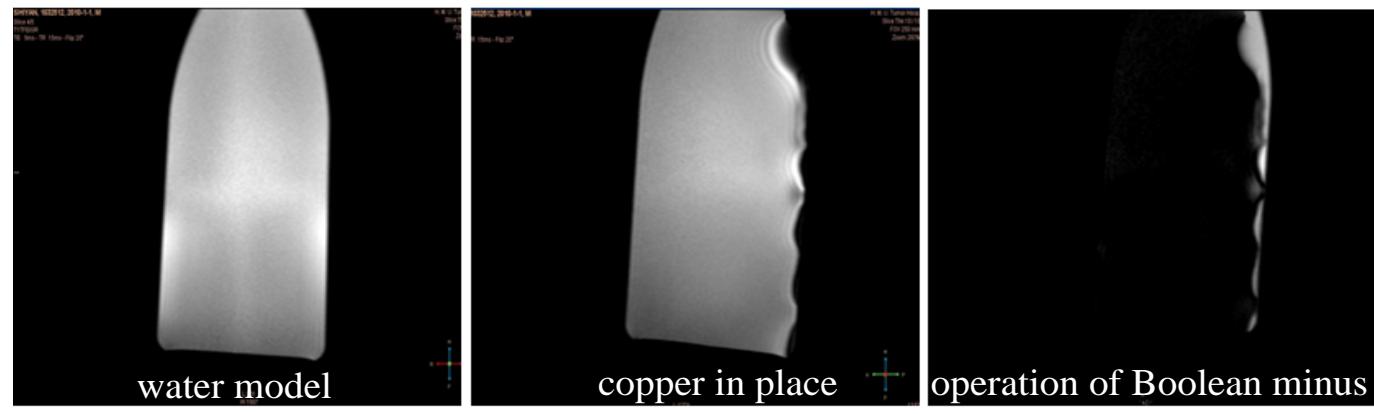

(a) Compatibility Experiment of Copper
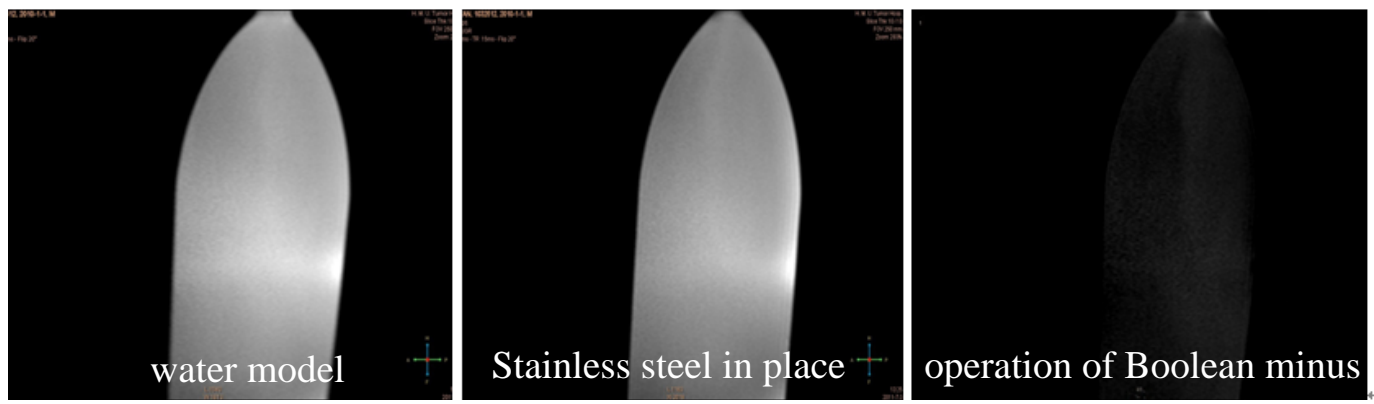

(b) Compatibility Experiment of Non-Magnetic Stainless Steel 

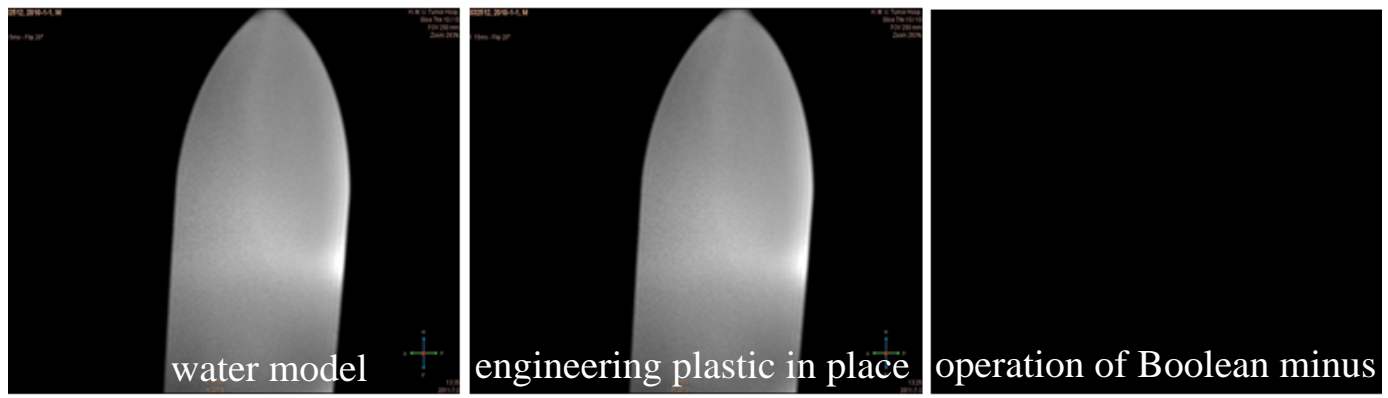

(c) Compatibility Experiment of Engineering Plastic

Figure 1. Compatibility Experiment of Materials

In this paper, non-magnetic stainless steels are selected as the materials of the screws, nuts, scissor lift, sliders, guides and biopsy needle. The auxiliary compression plates, moving plate, tissue slot and platform are made of nylon with high thermal conductivity and low density. The high-speed moving parts such as screw and bearing are made of polyformaldehyde (POM) with high rigidity and high hardness to avoid the generation of eddy currents.

\section{Analysis of Available Space}

A MRI scanner typically has a bore diameter of $550 \mathrm{~mm}$ to $700 \mathrm{~mm}$ [7]. Breast biopsy is typically performed with the patient in a prone position [8], that is, patient lies prone on an elevated patient couch, and the robot is designed to utilize the free space below the elevated patient couch, as shown in Figure 2. Figure 3 illustrates the spatial constraints inside an MR scanner by delineating the limits of a $600 \mathrm{~mm}$ bore gantry on transverse. Obviously the available space is very small, only about $420 \mathrm{~mm} \times 230 \mathrm{~mm}$.

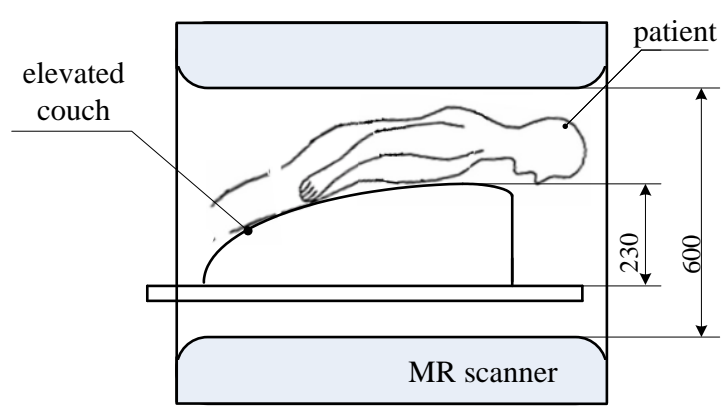

Figure 2. Prone Position of Patient in MR Scanner

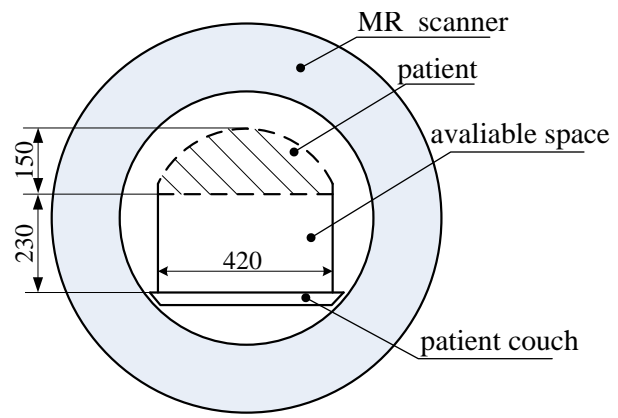

Figure 3. Illustration of Available Space

\section{Design of Robot}

The basic premise of the design of the robot is similar to existing designs [9-12], where the patient lies prone on an elevated surface attached to the patient couch. This surface has two openings through which the breasts can be placed. To overcome the aforementioned limitations of high field and limited available space, this paper proposes the following solution:

(1) An auxiliary positioning mechanism composed of a moving plate and a fixed plate is designed to reduce the movement of target tissue. Breast is stabilized by compressing it 
between the two plates. The robot is placed under the elevated surface to perform biopsy, as shown in Figure 4.

(2) Allowing for comfortable prone position of the patient, height curve of Z-axis of elevated patient couch is designed closed to logarithmic curve with base number greater than 1. The movement of the robot in $Z$ direction is performed by double scissor lift mechanism to efficiently utilize the space.

(3) The robot is remotely actuated by stepper motors mounted 1.5 meters away from the magnetic resonance scanner to avoid electrical interference on magnetic resonance imaging. The motors are connected to the shafts of robot via flexible shaft which can link two shafts of not on the same axis or not have the same direction, avoiding the generation of eddy current to affect the clarity of local image or cause image distortion.

Figure 5 illustrates the robot design, which resides all its components under the elevated patient couch. The robot has 5 DOF which consists of 3-DOF Cartesian motions $(X, Y, Z$, corresponding to the location of end effector with a needle attached), 1-DOF rotation around Y-axis $(\theta$, corresponding to the orientation of end effector) and 1-DOF insertion ( $d$, corresponding the depth of insertion along the desired path). In addition, the auxiliary positioning mechanism has one translational DOF along $\mathrm{X}$-axis ( $p$, corresponding to the immobilization of breast).

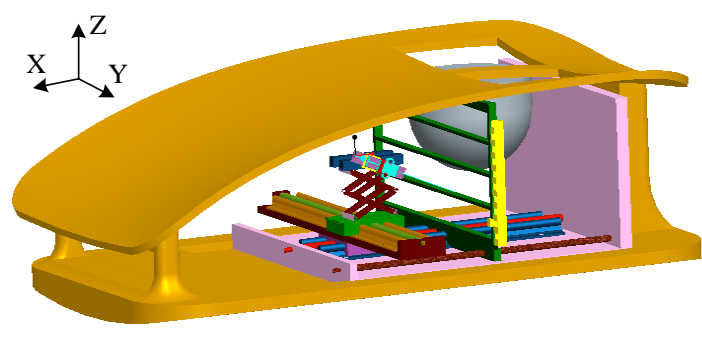

Figure 4. Structure of the Robot

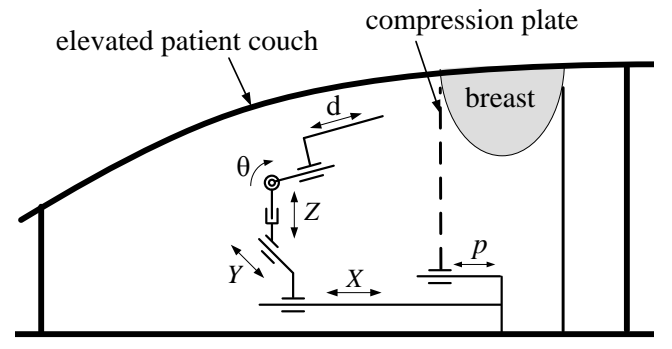

Figure 5. Illustration of the Robot

\subsection{Design of the Location Mechanism}

Location mechanism is a 3-DOF Cartesian positioner which provides three orthogonal DOF, $X, Y$ and $Z$, for the global location of end effector. The two horizontal DOF ( $X$ and $Y$ ) are actuated with lead screw, the mobile platform is constructed of circular guide with support. As mentioned above, the available space of $Z$ direction is very small. Furthermore, in order to satisfy the comfortable placement of the patient, the height of elevated patient couch in $Z$ direction changes nonlinearly, thus this paper adopts double scissor lift mechanism to realize the movement of the end effector in $Z$ direction, which can efficiently utilize the space of $Z$ direction. The structure sketch and 3D model of scissor lift mechanism are shown in Figure 6 . The left slider connected to the scissor lift are fixed with nut, when exerting torque $M$ on bar 1, driving force is generated on bar 2 and angle $\theta$ is changed, thus right slider will move along $X$ direction to accomplish the displacement of end effector along $Z$ direction.

It can be obtained from Figure 6 that

$$
H=4 h=2 L \sin \theta
$$

where, $L$ is the length of the bar, $H$ is the height of the scissor lift with the angle $\theta$, then the displacement $\Delta H$ along $Z$ direction is

$$
\Delta H=H_{1}-H=2 L\left(\sin \theta_{1}-\sin \theta\right)
$$

where, $H_{1}$ is the height of the scissor lift with the angle $\theta_{1}$. 


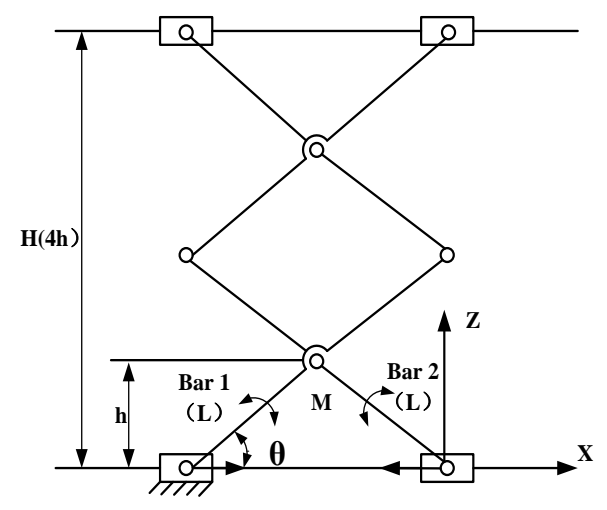

(a) Sketch of Scissor Lift Mechanism

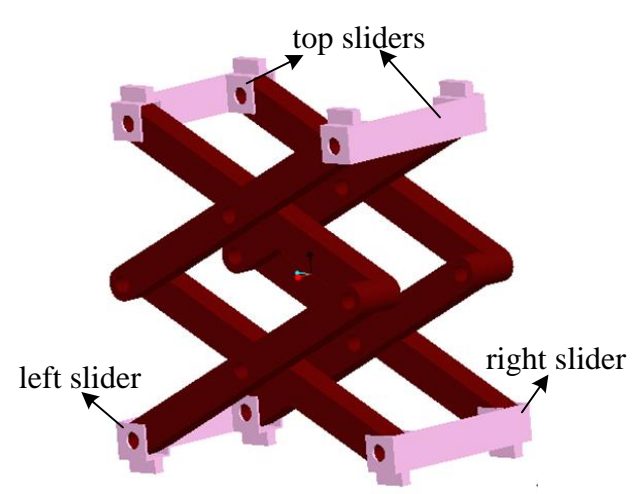

(b) 3D Model of Scissor Lift

Figure 6. Double Scissor Lift

\subsection{Design of the Orientation Mechanism}

The orientation mechanism has a rotation DOF around $Y$ axis to realize the swing of the end effector, as shown in Figure 7. The range of the swing angle is from $-60^{\circ}$ to $60^{\circ}$. To stabilize the breast, a moving plate with fenestrated grid is placed in front of the end effector. During the needle insertion, the target tissue may be obstructed by the moving plate. So a rotation DOF around $Y$ axis is very necessary to bypass the moving plate to achieve any target tissue.

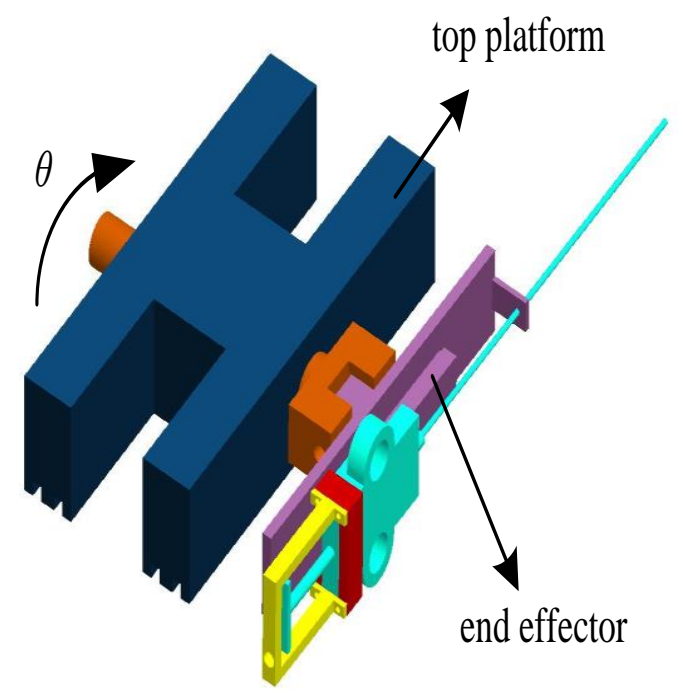

Figure 7. The Orientation Mechanism

\section{FEA of the Robot}

Finite Element Analysis (FEA) is used to simulate the tress and deformation of the breast biopsy robot. The parameters setting in FEA is shown in Table 1. 
Table 1. Parameters Setting in FEA

\begin{tabular}{ll}
\hline Items & \multicolumn{1}{c}{ Parameters } \\
\hline & $\begin{array}{l}\text { non-magnetic stainless steel: Poisson's ratio } 0.3, \text { density } 7.70 \mathrm{e}+3 \mathrm{~kg} / \mathrm{m}^{3} \text {, the elastic } \\
\text { materials }\end{array}$ \\
& modulus 2.0e+11 Pa \\
& Nylon: Poisson's ratio 0.3 , density $1.15 \mathrm{e}+3 \mathrm{~kg} / \mathrm{m} 3$, the elastic modulus $1.0 \mathrm{e}+9 \mathrm{~Pa}$ \\
& POM: Poisson's ratio 0.3, density $1.41 \mathrm{e}+2 \mathrm{~kg} / \mathrm{m} 3$, the elastic modulus $2.9 \mathrm{e}+9 \mathrm{~Pa}$ \\
loads & vertical pressure of needle tip: $0.32 \mathrm{Mpa}$ (tested by experiment) \\
\hline
\end{tabular}

\subsection{Total Statics Analysis of the Robot}

Figure 8 shows the stress nephogram of the robot. It can be seen that the stress with loads and constraints ranges from $2.89 \mathrm{e}-11$ to $0.95 \mathrm{Mpa}$, the maximum stress occurs at the boundary of the top platform and the top sliders. The top sliders play an important role of connecting the top platform and the scissor lift. The slider has a small size comprising a moving pair and a rotating pair, and has no chamfer processing, so stress concentration will produce, but the maximum stress is still within the permissible range of stress. We can fillet it or add stiffened panels to improve its strength.

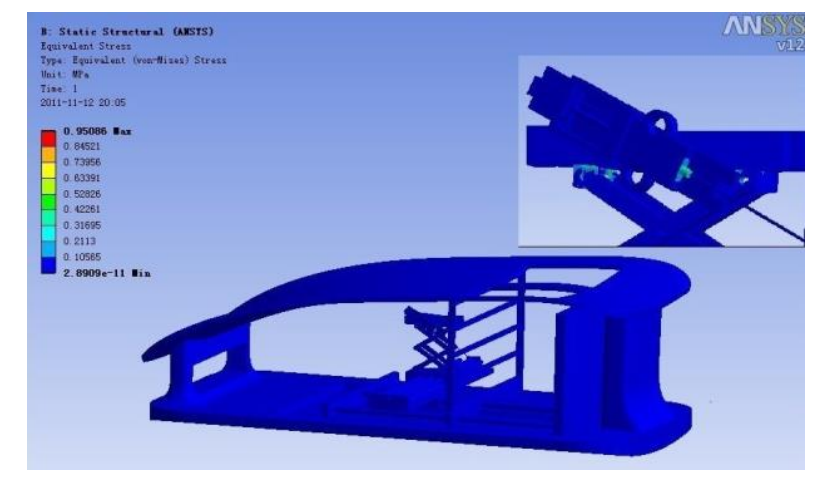

Figure 8. The Stress Nephogram of the robot

Figure 9 shows the deformation nephogram of the robot with loads. The deformation ranges from 0 to $0.6 \mathrm{e}-3 \mathrm{~mm}$, within the allowable range of error (The error for breast biopsy is $1 \mathrm{~mm}$.) Maximum deformation occurs at the end of the biopsy needle because the biopsy needle is long and thin, and it is directly affected by the stress. The robot has small size; most of materials are engineering plastics with low density, and scissor lift is made of nonmagnetic stainless steel which has high elastic modulus and good stiffness. Therefore, the total deformation of the robot becomes smaller, which can meet the precision requirement for breast biopsy. 


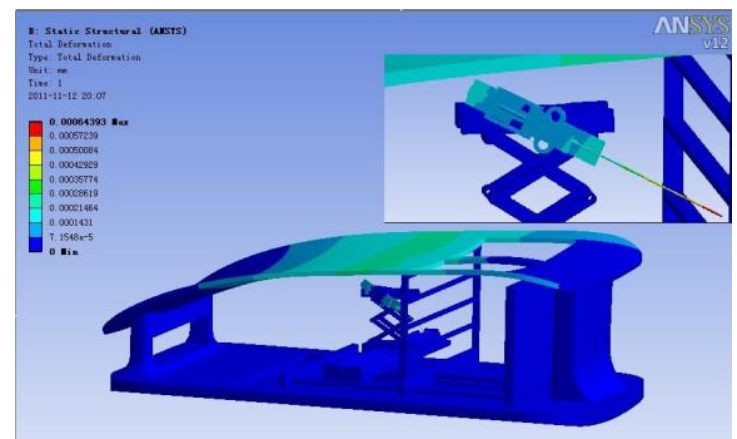

Figure 9. Total Deformation of the Robot

\subsection{Statics Analysis of the Top Slider}

It can be known from total statics analysis of the robot that the maximum stress and deformation generated at the top sliders and biopsy needle. The designs of the two parts are more crucial in this paper, so we implement static analysis for these two parts. Top sliders are important connections, at the ends of which there are four location holes used for pins connection. Therefore, the stress and deformation of top sliders will impact the total positioning precision of the robot. Figure 10 shows the deformation of top sliders. It can be seen that maximum deformation is $0.21 \mathrm{e}-3 \mathrm{~mm}$, which at the contact point between slider and top platform, because the connection has not been chamfered, so we need to fillet it or add stiffened panels to improve the structure and strengthen the mechanical properties of structure.

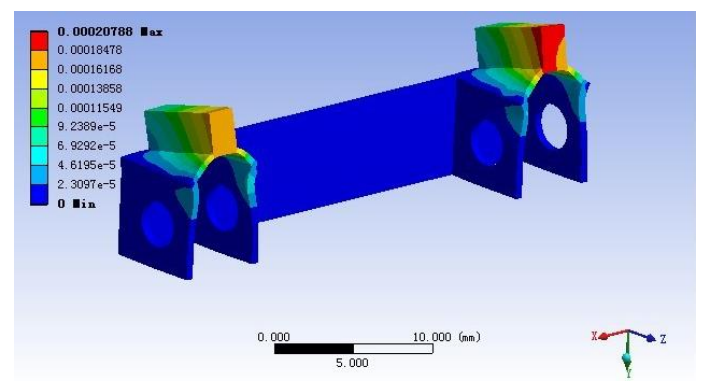

Figure 10. The Deformation of Top Slider

\subsection{Statics Analysis of the Needle Insertion Module}

Biopsy needle is made of non-magnetic stainless steel, with the outer diameter $2 \mathrm{~mm}$ and the length of $90 \mathrm{~mm}$, material of other parts are POM. The deformation of biopsy needle ranges from 0 to $0.77 \mathrm{e}-3 \mathrm{~mm}$, as shown in Figure 11. Biopsy needle is thin and long, we can put the needle as a cantilever beam in engineering mechanics. So the maximum deformation occurs at the end of needle, which directly affected by the stress. But the maximum deformation is still within the permissible range of error and meets the accuracy requirement for breast biopsy. 


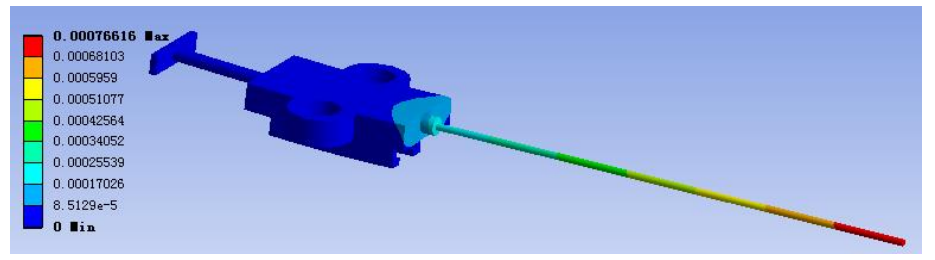

Figure 11. The Deformation of the Biopsy Needle

\section{Conclusions}

We designed an MRI-compatible robot for breast biopsy. The robot has 5 DOF that realize the location and orientation of its end-effector and needle insertion. The construction materials are selected for compatibility with the strong magnetic fields inside the MR scanner. The results of statics analysis demonstrate that the robot meet the requirement of stiffness and precision.

\section{Acknowledgements}

This work is supported in part by National Natural Science Foundation of China (No.51075105), by Outstanding Academic Leader Item of Harbin Technological Innovation Special Fund (No.2011RFXXS075), by Heilongjiang Province Education Bureau Project (Grant No. 12531122).

\section{References}

[1] World Health Statistics, [Online]. Available: http://www.who.int/whosis/whostat/EN_WHS08_ Part1.pdf, (2008).

[2] G. Vlastos and H. M. Verkooijen, "Minimally invasive approaches for diagnosis and treatment of early-stage breast cancer", Oncologist, vol. 12, no. 1, (2007), pp. 1-10.

[3] M. B. Piao, Y. L. Fu and S. G. Wang, "Development of Surgical Assistant Robots and Analysis of Key Technologies", Machinery Design \& Manufacture, no. 7, (2008), pp. 174-176.

[4] I. Bricault, N. Zemiti, E. Jouniaux, C. Fouard, E. Taillant, F. Dorandeu and P. Cinquin, "A Light Puncture Robot for CT and MRI Interventions", Engineering in Medicine and Biology Magazine, IEEE, vol. 27, no. 3, (2008), pp. 42-50.

[5] E. G. Christoforou, A. O zcan and N. V. Tsekos, "Manipulator for Magnetic Resonance Imaging Guided Interventions: Design, Prototype and Feasibility", Proceedings of the 2006 IEEE International Conference on Robotics and Automation, (2006), pp. 3838-3843.

[6] R. Kokes, K. Lister, R. Gullapalli, B. Zhang, H. Richard and J. P. Desai, "Towards a Teleoperated Needle Driver Robot with Haptic Feedback for RFA of Breast Tumors under Continuous MRI", Medical Image Analysis, vol. 13, no. 3, (2009), pp. 445-455.

[7] N. V. Tsekos, A. Khanicheh, E. Christoforou and C. Mavroidis, "Magnetic Resonance-Compatible Robotic and Mechatronics Systems for Image-Guided Interventions and Rehabilitation: A Review Study", Annu. Rev. Biomed. Eng., vol. 9, (2007), pp. 351-387.

[8] V. G. Mallapragada, N. Sarkar and T. K. Podder. "Toward a Robot-assisted Breast Intervention System", IEEE/ASME Transactions on Mechatronics, vol. 16, no. 6, (2011), pp. 1011-1020.

[9] E. Schneider, K. W. Rohlin, M. D. Schnall, R. O. Giaquinto, E. A. Morris and D. Ballon, "An Apparatus for MR-Guided Breast Lesion Localization and Core Biopsy: Design and Preliminary Results", Journal of Magnetic Resonance Imaging, vol. 14, (2001), pp. 243-253.

[10] Y. Yu, K. Van, T. Podder, W. S. Ng, K. Brill and L. Liao, "MRI-guided robot-assisted lumpectomy for surgical management of early breast cancer: Preliminary investigation and use case analysis", BioInformatics and BioEngineering, 8th IEEE International Conference on. IEEE, (2008), pp. 1-6.

[11] B. T. Larson, A. G. Erdman, N. V. Tsekos, E. Yacoub, P. V. Tsekos and I. G. Koutlas, "Design of an MRIcompatible robotic stereotactic device for minimally invasive interventions in the breast", Journal of biomechanical engineering, vol. 126, no. 4, (2004), pp. 458-465.

[12] M. Smith, X. Zhai, R. Harter, M. Elezaby and S. Fain, "A Novel MR-guided Interventional device for 3D circumferential Access to Breast tissue", Medical physics, vol. 35, no. 8, (2008), pp. 3779-3786. 